\title{
On the Engineering of Systems of Systems: key challenges for the requirements engineering community
}

\author{
Cornelius Ncube, \\ Software Systems Research Centre, \\ School of Design, Engineering \& Computing, \\ Bournemouth University, \\ Poole, Dorset, BH12 5BB, UK \\ cncube@,bournemouth.ac.uk
}

\begin{abstract}
Software intensive systems of the future will be ultra large-scale systems of systems. Systems of Systems Engineering focuses on the interoperation of many independent, self-contained constituent systems to achieve a global need. The scale and complexity of systems of systems posses unique challenges for the Requirements Engineering community. Current requirements engineering techniques are inadequate in addressing these challenges and new concepts, methods, techniques, tools and processes are required. This paper identifies some immediate key challenges for the Requirements Engineering community that need to be scoped and describes some roadmapping activities that aim to address these challenges.
\end{abstract}

Keywords-systems of systems, systems of systems engineering, requirements engineering, interoperation, emergent behaviour, complex systems

\section{SCIENTIFIC AND TECHNOLOGICAL BASIS}

Software-intensive systems of the future will be ultralarge-scale systems-of-systems [1, 2, 5]. Systems-of-Systems Engineering [4], is an emerging interdisciplinary approach that focuses on ultra-large scale interoperation of many independent, self-contained constituent systems in order to satisfy a global need $[1,2]$. Systems of Systems (SoS) are distinguished from very large but monolithic complex systems by (a) the operational independence of their constituent systems, i.e. each constituent system can operate independently and is capable of achieving its own goals in the absence of the other systems; (b) managerial independence of their constituent systems, i.e. the constituent systems are managed independently and can be added or removed from the System of Systems; (c) their evolutionary nature in which functions and purposes are added, removed or modified as needed, i.e. the SoS adapts to fulfill its (possibly evolving) objectives as its underlying technologies and needs evolve with time; (d) their emergent behaviors that cannot be localized to any constituent system, i.e. the functionality and behaviour of the SoS develops in ways not achieved by the individual systems, and (e) a geographical distribution that limits the interaction of the constituent systems to information exchange [3, 11]. Systems of Systems are further categorized [3] as either (a) directed, in which the interoperable system-of-systems is built to fulfill a specific purpose; (b) collaborative, in which constituent systems 'voluntarily' collaborate in an agnostic way to achieve an agreed-upon central purpose; (c) acknowledged
[12] in which there are recognized objectives, a designated manager and resources for the system of systems. However, the constituent systems return their independent ownership, objectives, funding, development and sustainment approaches and (d) virtual, in which there is no central agreed purpose for the system-of-systems [3]. Furthermore, the constituent systems can range from legacy or custombuilt systems, COTS-based systems, or Service-centric systems and must interoperate or integrate with a range of processes and policies $[1,6,8]$. In many cases, due to their large-scale and complexity, Systems of Systems cannot be designed; they emerge and grow spontaneously with no one organization able to control the whole system of systems. They are too large and/or too big to fail!

\section{THE SOCIETAL IMPACT OF SYSTEMS OF SYSTMS}

In May 2010 the whole of Northern Europe was transformed into a no-fly zone due to the Icelandic volcanic eruption. Thousands of travellers across Europe were stranded when the Air, Road, Rail, and Sea Transport Systems were unable to cope. This had a cascading impact as holiday resorts and hotels around the world were not able to cope with stranded passengers. According to a 'conservative estimate' by the International Air Transport Association, airlines were expected to lose over \$200 million a day in revenue.

In 2007 a liquidity shortfall in the United States banking system triggered a global financial crisis considered by many economists to be the worst financial crisis since the Great Depression of the 1930s. This resulted in the collapse of large financial institutions, the bail out of banks by national governments, and downturns in stock markets around the world. In many areas, the housing markets suffered, resulting in numerous evictions, foreclosures and prolonged vacancies. It contributed to the failure of key businesses, declines in consumer wealth estimated in the trillions of U.S. dollars, substantial financial commitments incurred by governments, and a significant decline in economic activity.

In their different ways the above two examples underline how low-probability events can have catastrophic impacts locally and across the world. Also the increasingly internalization of business, the emergence of regional and global environmental problems, planning the future 
resources of the planet, dealing with climate change demonstrate that taken as a whole, the societal impact of these types of complex and inter-connected Systems of Systems problems do not respect nor are constrained by national borders. Solving these types of problems will require involving many different disciplines such as social sciences, mechanical, manufacturing, systems engineering, civil engineering, control systems, computer science, management science, mathematics, psychology, sociology, ergonomics, finance, economics, urban planning, religion etc, that have evolved to possess remarkable resilience and stability in the face of ever changing, often hostile environments.

\section{OPERATIONAL ENVIRONMENT OF SYSTEMS OF SYSTEMS}

The scale, complexities and challenges presented by the emergence of systems-of-systems requires us to go beyond the traditional software systems engineering practices. Current software systems engineering methods and techniques have not prepared us to develop these types of systems of systems and our intuition offers little or no guidance. SoS problems often exhibit many of the characteristics of the so called "wicked problems" [14], i.e. problems that are extremely complex and not bounded or stable; they do not have unique, right solutions, but rather solutions that are either better or worse than others, and they do not have a definitive formulation. Also, whereas traditional Systems Engineering (SE) focuses on building the right system, Systems of Systems Engineering (SoSE) focuses on choosing the right systems and their interactions to satisfy the requirements. Thus, SoSE requires a different set of skills and, to a significant extent, involves different multiple stakeholders than from traditional systems engineering. In SoS there are stakeholders for both the SoS and for the constituent systems themselves. These stakeholder groups each have their own objectives and organizational context which form their expectation with respect to the SoS [12]. The stakeholders of the SoS may have limited knowledge of the constraints and development plans for the individual systems. Stakeholders of individual systems may have little interest in the SoS, may give SoS needs low priority or may resist SoS demands on their systems. These competing stakeholder interests establish the complex stakeholder environment for SoS systems engineer. Current requirements engineering methods can not handle these competing stakeholder demands [12].

Also in a single system, the operational objectives are established based on structured requirements along with defined concepts of operation and priorities [12]. On the other hand, a SoS is engineered to create operational capabilities that are beyond that which the constituent systems can provide independently. A SoS has requirements both at the level of the entity formed by the interoperating constituent systems and at the level of the individual constituent systems themselves. The requirements for the SoS are at a higher level and are defined in terms of capabilities rather atomic requirements statements as is in the traditional systems engineering. Because the requirements are often cast in terms of broader capability objectives, the SoS systems engineering team is required to engage with SoS managers, multiple stakeholders, and users to derive the SoS requirements from capability objectives and then address them using the functionality or capabilities of the constituent systems, augmented with enhancements or new development [12].

\section{KEY CHALLENGES FOR THE REQUIREMENTS ENGINEERING COMMUNITY}

There are three immediate areas of research that we think the RE community need to scope in the first instance: Interoperation (and composition as special case), Requirements Engineering and Emergent Behavior Management. Each is discussed in turn:

(a) Interoperation: systems of systems depend on distributed control, collaboration, interoperation, influence, emergent composition and other emergent behaviours as their primary mechanisms for achieving their purpose $[3,6]$. These global behaviours emerge from the cumulative actions and interactions of constituent systems that are then propagated throughout the system of systems [2,6 13]. Interoperation in the context of systems-of-systems is an agnostic cooperative process that uses distributed influence and trust to combine constituent systems that were separately developed to achieve a common purpose $[6,8]$. It is distinguished from composition which is a process that uses centralized control to combine constituent systems that were designed to work together in a predefined structure with known fixed roles for each constituent system [6]. A Requirements Oriented Interoperation Framework that uses influence and emergence requirements as a primary interoperation mechanism is therefore needed to organize and facilitate the interoparion of constituent systems in an agnostic fashion to achieve global needs [3, 6]. The Framework should identify key interoperation influencing requirements. It should define new interoperation requirements concepts including emergent composition, cascade effects, epidemics and emergence-induced orchestration as influence for achieving interoperation. New requirements development concepts and techniques associated with effective interoperation of systems- ofsystems that span organizational, platform, system management and geographical boundaries need to be developed to support the framework

(b) Requirements Engineering - The complexity of systems-of-systems represents a new reality that will require the requirements engineering community to change the way it thinks about current requirements engineering processes $[7,10]$. The nature and characteristics of requirements for systems-of-systems cuts across many desciplines, are 
fragmented, sometimes conflicting, unstable, unknowable and may not be fully defined [6, 10]. Also these requirements are defined as either systems-of-systems requirements which are properties of the overall system-ofsystems that are addressed using the capabilities of the constituent systems, or constituent system-level requirements which are allocated to particular constituent system(s). These types of requirements are constantly changing and this makes theier partitioning very difficult. This means the traditional concept of static, signed-of requirements is not suitable for systems-of-systems. New requirements engineering processes, management methods, techniques and tools that can dynamically respond to unstable, fragmented, continually changing requirements are needed. These methods and tools should not only be able to handle problems that are associated with influence requirements, cascades effects, epidemics but also be able to deal with problems associated with partitioning a large-scale system-of-systems into multiple autonomous independently evolving constituent systems. The tradeoffs required for this partitioning are critical for the successful development of systems-of-systems and therefore new requirements engineering philosophies and theories that exploit emergent behaviour and influence as a modelling mechanism to understand the uniqueness of requirements for systems-ofsystems will need to be developed. Particular emphasis will need to be placed on requirements engineering processes for: handling competing stakeholder demands, weaving interoperable systems-of-systems, persistant interoperable architecture, dynamic evolution of systems-of-systems and how emergent behaviours impact on requirements stability.

(c) Managing Emergent Behaviour - systems-ofsystems are characterized by emergent properties that are properties of the entire system not contained within or generated by any single system or a small group of systems [6]. These global behaviours emerge from the cumulative actions and interoperations of the constituent systems that are propagated throughout the system of systems $[2,6,13]$. Emergent composition is the means by which influences in the form of interactions between constituent systems are combined to generate global systems properties or behaviours that cannot be derived from individual systems $[3,6]$. Influence is the underlying mechanism by which constituent systems interact. Emergent behaviour arises from influence relationships through two primary mechanisms: cascade effects and emergent composition [6]. Cascade effects are the means by which influence and emergent effects are propagated throughout a system of systems. Cascade effects are successions of state changes in a sequence of constituent systems generated from a single initial influence. Cascades are critical to both the successes and failures of systems of systems therefore we need to understand their impact. Epidemics are special forms of cascade effects that are particularly appropriate for orchestration because unlike other cascade effects, they are able to influence a large number of constituent systems without having to direct information to particular systems. Just as in diseases, in an epidemic in systems-of-systems, the number of constituents systems that are influenced increases at each step. However, currently we do not understand how to model the key factors that stimulate these epidemics. Because these epidemic effects can produce negative consequences such as cascading failures, deadlocks, and communications problems there is a need to develop consequence and impact analysis techniques. However we currently don't understand enough about the processes by which constituent systems interact to produce emergent behaviour, influences, cascades and epidemic effects. Effective requirements engineering methods, tools and techniques for managing emergent effects with predictable results are required $[1,2,6,9]$.

\section{CONCLUSIONS AND FUTURE SYSTEMS OF SYSTEMS INITIATIVES}

The author (Ncube) and colleagues are currently involved on the following initiatives that aim to provide strategic research agendas and roadmaps for addressing some of these key challenges:

FP7: Trans-Atlantic Research and Education Agenda in Systems of Systems project: On $1^{\text {st }}$ September 2011, the author (Ncube) together with Henshaw (Loughborough University, UK), Jamshidi (University of Texas, San Antonio, USA) and DeLaurentis, (Purdue University, USA) will be starting a 2-year Trans-Atlantic Research and Education Agenda in Systems of Systems (T-AREA-SOS) research project funded by the EU FP7 programme. The project deals with the production of a broad-based EU-US agenda for socially-significant system-of-systems research, applicable across areas including energy, transport, health, emergence response, water management and manufacturing as exemplars. Key outputs of T-AREA-SOS will be a strategic research agenda and roadmap and concrete research initiatives between the EU and the US for FP7/ Horizon 2020 and other international research programmes to deliver outcomes that address societal needs using Engineering of SoS as a mainstream discipline for the management of large-scale complex systems. Particular emphasis will be paid to requirements engineering and interoperability challenges

System-of-Systems Engineering Network (SoSEN): in 2010 Ncube, Henshaw (Loughborough University, UK), Jamshidi (University Of Texas, San Antonio, USA) and DeLaurentis, (Purdue University, USA) brought together a large EU-US Systems of Systems Engineering Network that included partners drawn from key academia, industry, government and non-government organisations. The initiative attracted support from 33 academics from 24 leading UK universities, 13 international academics from 11 international universities and 12 major organizations including the UK MoD, US DoD, Australian DoD, IBM and BAE Systems just to name a few. The aim of the 
network was to scope a research strategy for systems of systems engineering with an initial focus on requirements engineering, interoperation, emergent management, development process and education.

IEEE Technical Committee on Systems of Systems The IEEE Technical Committee on Systems of Systems of which DeLaurentis and Henshaw are co-chairs is initiating a road mapping activity for systems of systems. The purpose of the roadmap is to provide a cohesive framework through which new research may be initiated by the IEEE community and to assist research funders in targeting funding to meet the priority needs of industry and academia to meet the challenges facing society. The planed roadmap for SoS research and development is based around five and ten year horizons. It will incorporate the views of a range of industrial sectors, to enable an expanded engagement of the SoSE community.

INCOSE knowledge network in Systems of Systems The INCOSE Architectures Working Group has initiated a knowledge network in Systems of Systems led by DeLaurentis and Henshaw. This initiative is linked to the IEEE Technical Committee on Systems of Systems and will extend the range of experts contributing to the roadmap, especially those in industry

ICSoSE (International Consortium in Systems of Systems Engineering) Since 2007, an international effort led by DeLaurentis took place in US, UK, Australia, and many other nations to form a joint effort in meeting the challenges of integrated interoperable systems. The ultimate objective of this effort is to enhance the ability of SoS community members worldwide to address many new and challenging problems facing the community across a diverse set of problem domain applications. Since 2007, ICSoSE has conducted workshops and tutorials in Australia, France, China, India, Canada, Spain, UK, Finland, and Czech Republic

\section{US-SoSEN - US System of Systems Engineering Network}

Since early 2010 in the US, Jamshidi has led a national effort to create a US System of Systems Engineering Network (US-SoSEN) to examine the problems, and solutions strategies, associated with SoSE in various facets of America' s Education, National Defense, Homeland security, Energy needs, Space exploration, Environmental sustainability, Economy, Healthcare, Finances, and Manufacturing. It is expected the network will lead efforts in clarifying ambiguities and in seeking remedies to numerous open questions with respect to complex systems analysis, complex systems Engineering (CSE) that will educate and train America' s future "Complex systems engineers". The mission of this network is envisioned to (i) expedite transfer of knowledge and technology of CSE as a neutral party (ii) provide a forum to put forth calls to action in CSE, (iii) establish a community of interest to recommend set of solutions, (iv) Provide a forum to educate American graduate students in CSE for future needs of US, (v) Conduct collaborative research team from Network members and (vi) Cooperate with industry and governmental partners in CSE. The network has support from the IEEE and INCOSE.

\section{ACKNOWLEDGEMENT}

The author would like to acknowledge partners on the EU FP7 funded T-AREA-SOS project, Henshaw, Jamshidi \& DeLaurentis for their contributions in the various initiatives that aim to provide strategic research agendas and roadmaps for addressing some of the key Systems of Systems engineering challenges.

\section{REFERENCES}

[1] LSCITS - Large-Scale Complex IT Systems- an EPSRC Funded Programme, 2007, http://www.lscits.org/index.shtml

[2] Linda Northrop, Ultra-Large-Scale Systems, The Software Challenge of the Future, Software Engineering Institute, Carnegie Mellon University, June 2006 www.sei.cmu.edu/uls/

[3] Maier, M.W., "Architecting Principles for System of Systems," Systems Engineering, Vol. 1, No. 4, 1998, pp. 267154

[4] Jamshidi, M., "System-of-Systems Engineering - A Definition," IEEE SMC 2005, 10-12 Oct. 2005.

[5] Ian Sommerville, Software Engineering: Challenges for the $21^{\text {st }}$ Century, ICCBSS 2008, Madrid

[6] David A. Fisher, An Emergent Perspective on Interoperation in Systems of Systems, TECHNICAL REPORT, CMU/SEI2006-TR-003, ESC-TR-2006-003, March 2006

[7] B. Craig Meyers, James D. Smith, Peter Capell, Patrick R. H. Place, Requirements Management in a System-of-Systems Context: A Workshop, CMU/SEI-2006-TN-015, March 2006

[8] Lisa Brownsword, David Fisher, Ed Morris, James Smith, Patrick Kirwan, System-of-Systems Navigator: An Approach for Managing System-of-Systems Interoperability, Technical Note, CMU/SEI-2006-TN-019, April 2006

[9] Jeremy Kaplan, Challenges and Approaches to System of Systems Engineering, Industrial College of the Armed Forces, June 14, 2005

[10] Steve Easterbrook, Scale Changes Everything: Understanding the Requirements for Systems of Systems, ICCBSS 07, February 26 - March 2, 2007, Banff, Canada

[11] A Report of a workshop on Systems of Systems Contribution to ICT Work Programme 2011+, DG INFSO G3, Brussels, September $21^{\text {st }} 2009$

[12] Systems Engineering Guide for Systems of Systems, US DoD, Version 1.0, August 2008

[13] Saeid Nahavandi, Modelling of Large Complex System from System of Systems Perspective, 2007, IEEE SoSE Conference, San Antonio, Texas, USA, April 16-18, 2007.

[14] Rittel, Horst, and Melvin Webber; "Dilemmas in a General Theory of Planning," pp. 155-169, Policy Sciences, Vol. 4, Elsevier Scientific Publishing Company, Inc., Amsterdam, 1973. 